\title{
Autologous Ex Vivo Lentiviral Gene Therapy for Adenosine Deaminase Deficiency
}

\author{
D.B. Kohn, C. Booth, K.L. Shaw, J. Xu-Bayford, E. Garabedian, V. Trevisan, \\ D.A. Carbonaro-Sarracino, K. Soni, D. Terrazas, K. Snell, A. Ikeda, D. Leon-Rico, \\ T.B. Moore, K.F. Buckland, A.J. Shah, K.C. Gilmour, S. De Oliveira, C. Rivat, \\ G.M. Crooks, N. Izotova, J. Tse, S. Adams, S. Shupien, H. Ricketts, A. Davila, \\ C. Uzowuru, A. Icreverzi, P. Barman, B. Campo Fernandez, R.P. Hollis, \\ M. Coronel, A. Yu, K.M. Chun, C.E. Casas, R. Zhang, S. Arduini, F. Lynn, \\ M. Kudari, A. Spezzi, M. Zahn, R. Heimke, I. Labik, R. Parrott, R.H. Buckley, \\ L. Reeves, K. Cornetta, R. Sokolic, M. Hershfield, M. Schmidt, F. Candotti, \\ H.L. Malech, A.J. Thrasher, and H.B. Gaspar
}

ABSTRACT

\section{BACKGROUND}

Severe combined immunodeficiency due to adenosine deaminase (ADA) deficiency (ADA-SCID) is a rare and life-threatening primary immunodeficiency.

\section{METHODS}

We treated 50 patients with ADA-SCID (30 in the United States and 20 in the United Kingdom) with an investigational gene therapy composed of autologous CD34+ hematopoietic stem and progenitor cells (HSPCs) transduced ex vivo with a self-inactivating lentiviral vector encoding human ADA. Data from the two U.S. studies (in which fresh and cryopreserved formulations were used) at 24 months of follow-up were analyzed alongside data from the U.K. study (in which a fresh formulation was used) at 36 months of follow-up.

\section{RESULTS}

Overall survival was $100 \%$ in all studies up to 24 and 36 months. Event-free survival (in the absence of reinitiation of enzyme-replacement therapy or rescue allogeneic hematopoietic stem-cell transplantation) was $97 \%$ (U.S. studies) and $100 \%$ (U.K. study) at 12 months; 97\% and 95\%, respectively, at 24 months; and 95\% (U.K. study) at 36 months. Engraftment of genetically modified HSPCs persisted in 29 of 30 patients in the U.S. studies and in 19 of 20 patients in the U.K. study. Patients had sustained metabolic detoxification and normalization of ADA activity levels. Immune reconstitution was robust, with $90 \%$ of the patients in the U.S. studies and $100 \%$ of those in the U.K. study discontinuing immunoglobulin-replacement therapy by 24 months and 36 months, respectively. No evidence of monoclonal expansion, leukoproliferative complications, or emergence of replication-competent lentivirus was noted, and no events of autoimmunity or graft-versus-host disease occurred. Most adverse events were of low grade.

CONCLUSIONS

Treatment of ADA-SCID with ex vivo lentiviral HSPC gene therapy resulted in high overall and event-free survival with sustained ADA expression, metabolic correction, and functional immune reconstitution. (Funded by the National Institutes of Health and others; ClinicalTrials.gov numbers, NCT01852071, NCT02999984, and NCT01380990.)
The authors' full names, academic degrees, and affiliations are listed in the Appendix. Address reprint requests to Dr. Kohn at the Department of Microbiology, Immunology, and Molecular Genetics, University of California, Los Angeles, 3163 Terasaki Life Science Bldg., 610 Charles E. Young Dr. South, Los Angeles, CA 90095, or at dkohn1@mednet.ucla.edu.

Drs. Kohn and Booth contributed equally to this article.

This article was published on May 11, 2021, at NEJM.org.

DOI: 10.1056/NEJMoa2027675

Copyright (๑) 2021 Massachusetts Medical Society. 
EVERE COMBINED IMMUNODEFICIENCY due to adenosine deaminase (ADA) deficiency (ADA-SCID) is an inborn error of metabolism that results in the accumulation of adenosine and deoxyadenosine, ${ }^{1}$ inhibition of DNA synthesis and repair, and substantial abnormalities of thymocyte development. ${ }^{2}$ Patients with ADA-SCID have profound lymphocytopenia, impaired immune function, and failure to thrive and, without treatment, die from infections by the age of 2 years. ${ }^{3}$

Expert guidelines recommend that patients initially receive enzyme-replacement therapy with pegylated ADA (PEG-ADA).,5 However, because long-term enzyme-replacement therapy does not provide full immune reconstitution, ${ }^{2,6,7}$ allogeneic hematopoietic stem-cell transplantation $(\text { HSCT })^{5}$ or autologous hematopoietic stem-cell gene therapy is recommended as more definitive treatment. ${ }^{4}$

Ideally, HSCT is performed with cells from an HLA-matched, related donor, but such donors are available for only approximately $20 \%$ of patients. ${ }^{2,8}$ The main risk of HSCT is graft-versushost disease, which is typically managed with potent immunosuppressive medications, thereby increasing the risk of infection and complications. ${ }^{9,10}$

An alternative treatment, ex vivo autologous hematopoietic stem-cell gene therapy with a $\gamma$-retroviral vector, ${ }^{11,12}$ was approved in Europe in 2016. ${ }^{13}$ However, in clinical trials involving patients with primary immunodeficiencies, the use of $\gamma$-retroviral vectors has resulted in vectorrelated leukemia and myelodysplastic events caused by insertional oncogenesis. ${ }^{14-16}$ Recently, a patient with ADA-SCID who was treated with a $\gamma$-retroviral vector gene therapy (in clinical development at the time of treatment) was found to have lymphoid T-cell leukemia approximately 4.7 years after the gene therapy. ${ }^{17}$

We developed a self-inactivating lentiviral vector, EFS-ADA LV, in which the long-terminal-repeat enhancer-promoter has been deleted and an internal "shortened" human elongation factor $1 \alpha$ gene promoter has been inserted to drive expression of the codon-optimized ADA complementary DNA transgene. ${ }^{18,19}$ This promoter has substantially less transactivation potential than $\gamma$-retroviral promoters. ${ }^{18,20,21}$ In this study, we evaluated the safety and efficacy of an investigational lentiviral gene therapy composed of au- tologous CD34+ hematopoietic stem and progenitor cells (HSPCs) that had been genetically modified ex vivo with EFS-ADA LV.

\section{METHODS}

\section{STUDY DESIGN AND OVERSIGHT}

We conducted an integrated analysis of data from two prospective, nonrandomized, phase 1-2 clinical studies conducted in the United States and a separate analysis of a nonrandomized, prospective, phase 1-2 clinical study conducted in the United Kingdom to assess the safety and efficacy of an autologous investigational lentiviral vector-mediated gene therapy composed of CD34+ HSPCs genetically modified ex vivo with EFS-ADA LV in patients with ADA-SCID. Details of the vector have been published previously, ${ }^{18}$ and descriptions are provided in the Supplementary Methods section and Fig. S1 in the Supplementary Appendix, available with the full text of this article at NEJM.org. Patients in the U.S. studies were treated at the University of California, Los Angeles, and the National Institutes of Health, Bethesda, Maryland, between 2013 and 2017, and patients in the U.K. study were treated at Great Ormond Street Hospital, London, between 2012 and 2016.

The studies were designed by academic authors and employees of the commercial sponsors. The studies were conducted in compliance with the independent ethics committees and institutional review boards, informed consent regulations, the Declaration of Helsinki, and the International Council for Harmonisation Good Clinical Practice Guidelines.

Orphan Drug and Breakthrough Therapy designations were granted by the Food and Drug Administration (FDA) for the investigational lentiviral gene therapy used in the U.S. studies (OTL-101) in October 2014 and August 2015, respectively. In August 2017, OTL-101 was granted a Promising Innovative Medicine Designation by the U.K. Medicines and Healthcare Products Regulatory Agency.

Clinical data were gathered by the authors during the treatment procedures and follow-up assessments. Data were analyzed in collaboration with the Orchard Therapeutics clinical and statistics teams, and the statistical analysis was performed by Phastar. The authors had access to the data, contributed to data interpretation and 
statistical reports, and take responsibility for the data integrity, accuracy, and analysis, as well as for adherence of the studies to the protocols, available at NEJM.org. Deidentified clinical data were transmitted to the sponsor without any associated private health information.

Initial drafts of the manuscript that was submitted were written by the first two authors with editorial support, based on authors' direction, provided by Comradis and paid for by Orchard Therapeutics. The authors edited and approved the manuscript for submission and publication.

\section{PATIENTS}

Children with a diagnosis of ADA-SCID who were 1 month of age or older and who lacked an HLA-matched sibling (or a related donor [in the U.S. study in which a cryopreserved formulation was used]) were eligible for enrollment in one of the two U.S. studies; a confirmed diagnosis of ADA-SCID, either an age younger than 5 years or an age of 5 to 15 years with preserved thymic function, and absence of a matched related donor for HSCT were required for participation in the U.K. study. The full list of inclusion and exclusion criteria is provided in Table S1. Ten patients in the U.K. study received EFS-ADA LVtransduced CD34+ HSPCs as part of a clinical study, and 10 were treated through the compassionate-use program under a Great Ormond Street Hospital "Specials" manufacturing license. Patients who were treated in the context of the compassionate-use program were enrolled either before or after the study because they required timely treatment as a result of clinical need, did not meet the inclusion criteria or met exclusion criteria, received the product through a different process, or were to receive a duration of enzyme-replacement therapy other than the duration defined in the study protocol. Written informed consent was obtained from the patients or their guardians in accordance with guidelines of the local independent ethics committee or institutional review board.

\section{STUDY PROCEDURES}

\section{Cell-Product Manufacture and Transplant}

In the U.S. studies, CD34+ HSPCs from bone marrow were enriched for transduction with EFS-ADA LV. In the U.K. study, CD34+ HSPCs were obtained from patients either by bone marrow harvest ( 3 patients) or with the use of mo- bilized peripheral blood (17 patients). The minimum target yield was at least $1 \times 10^{6} \mathrm{CD} 34+$ cells per kilogram of body weight (in the U.S. study in which a fresh formulation was used), $2 \times 10^{6}$ CD34+ cells per kilogram (in the U.S. study in which a cryopreserved formulation was used), and $4 \times 10^{6} \mathrm{CD} 34+$ cells per kilogram (in the U.K. study) (see the Supplementary Methods). In the U.S. studies, manufacturing exceptions were approved by the data and safety monitoring board, institutional review boards, and the FDA.

All the patients received nonmyeloablative weight-adjusted busulfan conditioning after stemcell harvest (see the Supplementary Methods). After enrichment, CD34+ HSPCs were transduced with EFS-ADA LV and evaluated for sterility, cell count, and viability before intravenous infusion. In the U.S. study in which a cryopreserved formulation was used, transduced HSPCs were cryopreserved before being thawed for infusion. All the patients were monitored for safety measures throughout conditioning, infusion of transduced HSPCs, and hematologic reconstitution. Patients received PEG-ADA enzyme-replacement therapy from the time of diagnosis until 30 days after treatment.

\section{Clinical Monitoring and Follow-Up}

Patients attended regular follow-up visits at which blood samples were obtained. Patients were monitored for adverse events, and biochemical and molecular tests, vector copy-number determinations, and vector-integration site analyses were performed (see the Supplementary Methods).

\section{END POINTS}

The safety end-point measures were the incidence and grade of adverse events and serious adverse events, including detection of replicationcompetent lentivirus or clonal expansion. Results of vector-integration site analysis were considered abnormal when a single site constituted more than $30 \%$ of the total integrations on two separate occasions. The primary efficacy end points were overall survival and event-free survival at 12 months and engraftment and the resulting immunologic and metabolic effects. Event-free survival was defined as survival in the absence of reinitiation of PEG-ADA enzymereplacement therapy or rescue allogeneic HSCT. Key secondary end-point measures included over- 
all survival and event-free survival at 24 months (all studies) and 36 months (U.K. study only) and measures of the engraftment of genetically modified HSPCs, ADA enzyme activity and deoxyadenosine metabolite detoxification, immune reconstitution, and infection. Infections of interest were severe infections and opportunistic infectious episodes, defined as infections leading to or prolonging hospitalization and document- ed infections with opportunistic pathogens, respectively. (A complete list of end points is provided in Table S3.)

\section{STATISTICAL ANALYSIS}

Overall survival and event-free survival were calculated and are presented as Kaplan-Meier curves for the time to death or an event at each study location. The safety population included all treat-

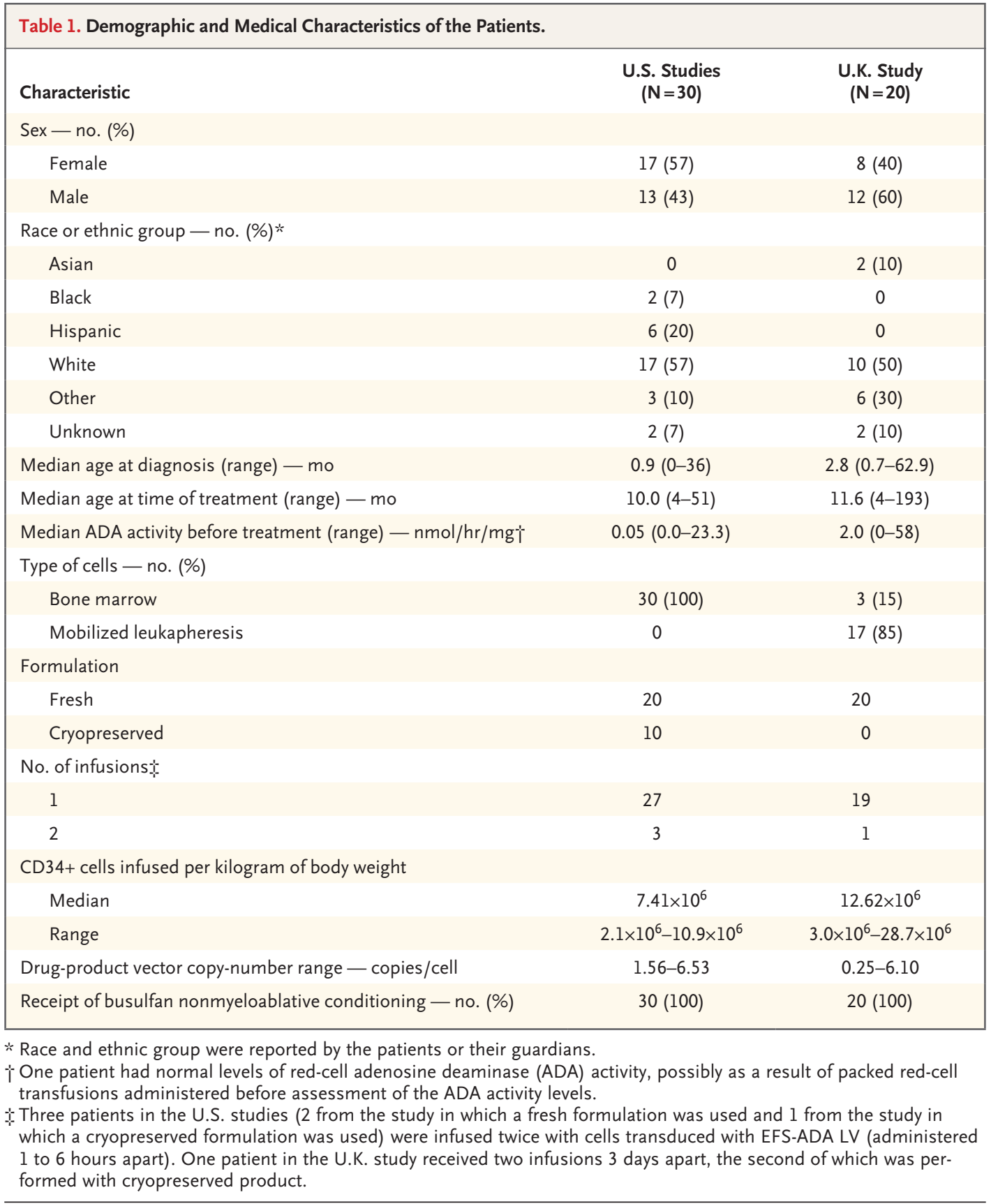


ed patients. Safety was analyzed descriptively over a period of 24 months (U.S. studies) or 36 months (U.K. study).

\section{RESULTS}

\section{BASELINE CHARACTERISTICS}

In total, 50 patients with ADA-SCID were treated with gene therapy across the three studies 30 in the United States (median age, 10.0 months; range, 4 to 51) and 20 in the United Kingdom (10 enrolled in the study and 10 treated through the compassionate-use program; median age, 11.6 months; range, 4 to 193) (Table 1). In the U.S. studies, 20 patients received the fresh formulation and 10 received the cryopreserved formulation. All the patients in the U.K. study received the fresh formulation (Table 1 and Table S2).

All the patients in the U.S. studies and 19 of the 20 patients in the U.K. study were receiving enzyme-replacement therapy at the time of treatment, and all patients were receiving prophylactic antimicrobials at screening. A total of 19 of 20 patients in the U.K. study and 29 of 30 patients in the U.S. studies were receiving immunoglobulin-replacement therapy at screening; the remaining patient started immunoglobulinreplacement therapy after infusion. Medical histories were consistent with conditions observed in children with ADA-SCID and were reflective of severe immunologic disease and sequelae of repeated infections. At the time of analysis, all the patients had completed 24 months (U.S.) or 36 months (U.K.) of follow-up, with the exception of 1 U.S. patient and 1 U.K. patient who were withdrawn because of a lack of sustained engraftment at 5.9 months and 12.2 months after treatment, respectively.

\section{SAFETY}

Among all the patients, no events of monoclonal expansion, leukoproliferative complications, emergence of replication-competent lentivirus, or deaths were noted. The frequencies of the 10 most prominent integration sites detected for each patient in peripheral blood mononuclear cell (PBMC) DNA at different time points are shown in Figure S2.

Adverse events were reported in all patients; most of the events were mild or moderate and were considered related to conditioning. During
24 months of follow-up in the U.S. studies, 73 of 421 adverse events were of grade 3 or 4 , two of which (leukopenia and neutropenia) led to the withdrawal of one patient in whom the transduced HSPCs did not engraft. Twelve patients in the U.S. studies had one or more serious adverse events, most frequently infections (27\%) and gastrointestinal events (17\%), only one of which was considered by the investigators to be treatment-related (staphylococcal bacteremia due to contamination of the fresh product, which resolved on day 3 without sequelae after antibiotic treatment). During 36 months of follow-up in the U.K. study, 60 of 321 adverse events were moderate to severe and 25 were serious (occurring in $55 \%$ of patients [11 of 20]), the most common being pyrexia (in 30\% of patients). One treatment-related serious adverse event was due to known drug-product contamination (staphylococcus was identified in harvested HSPCs; the patient received intravenous antibiotics immediately after infusion, and the patient's condition remained clinically stable).

Two patients in the U.S. studies and two patients in the U.K. study had serious adverse events of immune reconstitution inflammatory

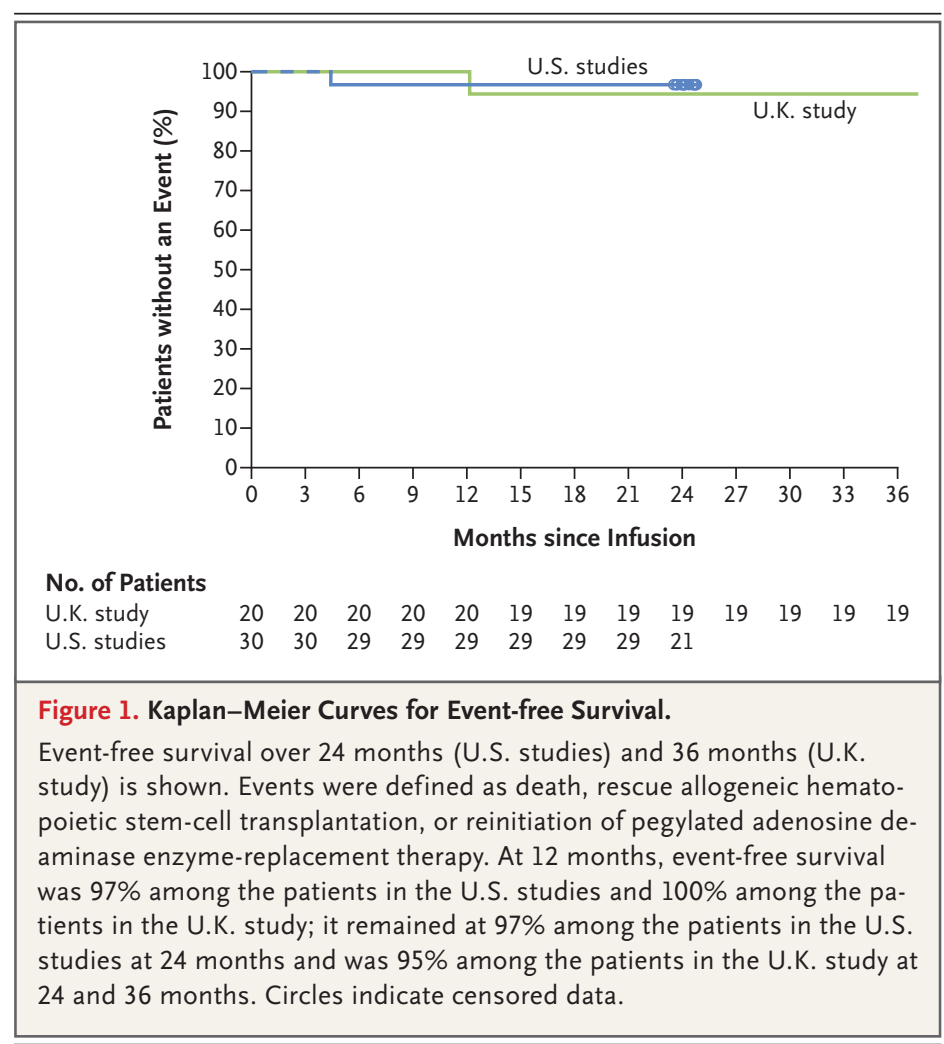


syndrome, beginning approximately 3 and 14 months after infusion in the U.S. studies and 3 and 22 months after infusion in the U.K. study; events were characterized by episodes of rash ( 2 of 2 U.S. patients and 1 of 2 U.K. patients), fever, and elevated inflammatory markers. One patient in one of the U.S. studies was infected with an atypical mycobacterium at initial diagnosis, for which treatment was ongoing, and 1 patient in the U.K. study had a preexisting bacille

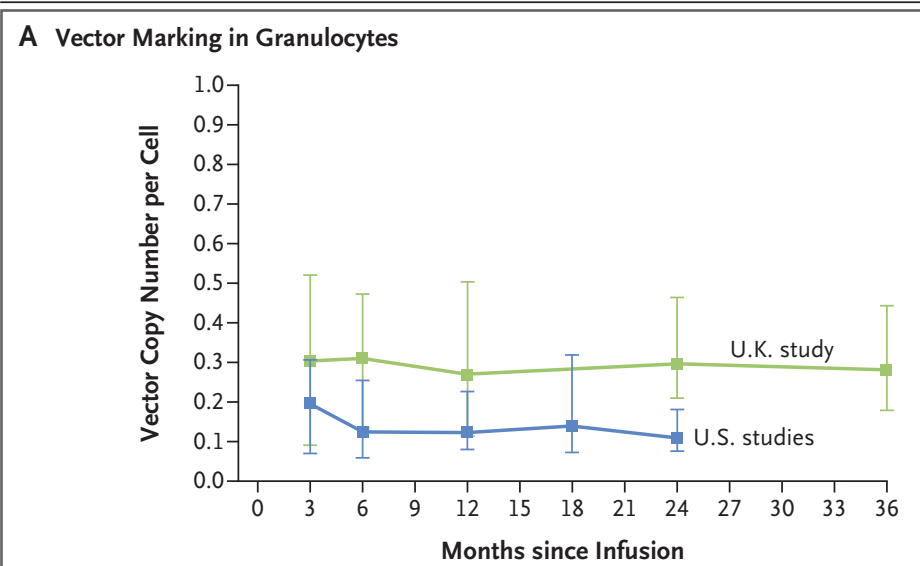

No. of Patients

U.K. study

U.S. studies

B Vector Marking in PBMCs

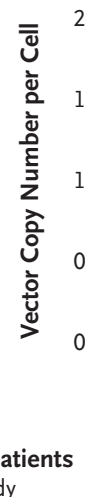

U.K. study
U.S. studies
$15 \quad 18$

$27 \quad 26$
18

27
18

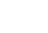

Figure 2. Median Vector Gene Marking in Granulocytes and PBMCs.

Vector copy number was determined by quantitative polymerase chain reaction (real-time or digital) with DNA extracted from fractionated peripheral blood with the use of primers and a probe specific for the human adenosine deaminase complementary DNA spanning exons 6 and 7. Panel A shows the median vector copy number in a granulocyte fraction enriched through immunomagnetic depletion with antibodies to CD3, CD19, and CD56. Panel B shows the median vector copy number in peripheral blood mononuclear cells (PBMCs). In both panels, I bars indicate interquartile ranges. Values of 0 are plotted as 0.001 .
Calmette-Guérin infection. All events were considered unrelated to gene therapy and resolved with supportive therapy, with all linked to transitory immune dysregulation during immune reconstitution. No autoimmune events or cases of graft-versus-host disease were noted in association with this investigational gene therapy.

\section{EFFICACY}

Across all studies, overall survival was $100 \%$ at 12 months and remained at $100 \%$ at 24 months for patients in all three studies; overall survival was still $100 \%$ at 36 months among the patients in the U.K. study (Fig. S3). At 12 months, eventfree survival was $97 \%$ among the patients in the U.S. studies and was $100 \%$ among the patients in the U.K. study (Fig. 1). Event-free survival remained at $97 \%$ among the patients in the U.S. studies at 24 months and 95\% among the patients in the U.K. study at 24 and 36 months. Reinitiation of enzyme-replacement therapy occurred in one patient in one of the U.S. studies; this patient was withdrawn from the study at 5.9 months after infusion because of a lack of engraftment and later received rescue HSCT. Enzyme-replacement therapy was reinitiated in one patient in the U.K. study at 12.2 months after treatment because of insufficient engraftment resulting in low ADA activity levels. Details of event-free survival and rescue treatment are provided in Table S4.

On the basis of vector gene marking, which was measured through determination of vector copy number, genetically modified granulocytes and PBMCs were detectable by month 3 in all patients with the exception of one patient in one of the U.S. studies, in whom engraftment of gene-modified cells had not been achieved. In one of the patients in the U.K. study, genetically modified granulocytes and PBMCs were detectable at month 3 but subsequently declined over time. In the patients with successful engraftment, the vector copy number was sustained in granulocytes through the end of follow-up. In PBMCs, the vector copy number increased through month 24 in both studies and was sustained through month 36 in the U.K. study (Fig. 2A and 2B). Similarly, in patients in the U.K. study, persistent gene marking was observed in $\mathrm{CD} 3+$ $\mathrm{T}$ cells and CD19+ B cells, increasing over 24 months and remaining stable up to 36 months (data not shown). 
In all three studies, the median ADA enzyme activity in red cells increased sharply during the first 3 months and remained within or above levels observed in healthy children at the last follow-up visit (Fig. S4A and S4B). ${ }^{22}$ In the U.S. studies, the median total deoxyadenosine nucleotide levels in red cells remained well below the maximum threshold indicating adequate detoxification $\left(0.1 \mu \mathrm{mol}\right.$ per milliliter $\left.{ }^{11,12,23}\right)$ until the end of the studies. Similarly, in the U.K. study, median levels of deoxyadenosine triphosphate remained below the maximum threshold indicating adequate detoxification ${ }^{12,24}$ through 36 months after treatment (Fig. 3).

At the final follow-up visit, lymphocyte counts in most patients had reached or come close to reaching the expected normal ranges for age for most lymphocyte populations. Median T-cell and T-cell subset counts decreased after conditioning and withdrawal of enzyme-replacement therapy but began to recover at month 3, with increases sustained through month 24 in the U.S. studies and through month 36 in the U.K. study (Fig. 4A through 4E). Institutional T-cell-count thresholds for stopping prophylactic antibiotic treatment (CD4+ cell count $>200$ per cubic millimeter [U.S. studies] ${ }^{25}$ or $>300$ per cubic millimeter [U.K. study]) were met by the majority of patients. In addition, the median naive T-cell counts increased to levels approximately 5 times above baseline by the end of both U.S. studies and the U.K. study, which suggests restoration of thymopoiesis. Median T-cell-receptor excision circle counts increased after treatment in the U.K. study and were sustained in both U.S. studies and the U.K. study up to month 24 and month 36 , respectively, which suggests that thymic output and de novo generation of naive $\mathrm{T}$ cells continued even after discontinuation of enzyme-replacement therapy (Fig. S5). Analysis of the T-cell receptor $\mathrm{V} \beta$ repertoire showed sustained clonal diversity in most patients (data not shown).

Median CD19+ B-cell and natural killer cell counts decreased to below baseline after treatment, as expected as a result of conditioning and cessation of enzyme-replacement therapy, but returned to approximately baseline levels over time (Fig. 4F and 4G). Reflective of improvements in B-cell function, the majority of patients had increases in serum IgM and IgA levels to within or above normal limits over time (Fig.
S6A and S6B). Furthermore, $90 \%$ of U.S. patients and $100 \%$ of U.K. patients discontinued immunoglobulin-replacement therapy by month 24 and month 36, respectively. Median IgG levels remained high after cessation of immunoglobulin replacement therapy (Fig. S6C). Among the patients who received a tetanus vaccination, 4 of 4 (100\%) in the U.S. studies and 13 of 14 (93\%) in the U.K. study had normal antibody responses when tested at least 3 months after cessation of immunoglobulin-replacement therapy.

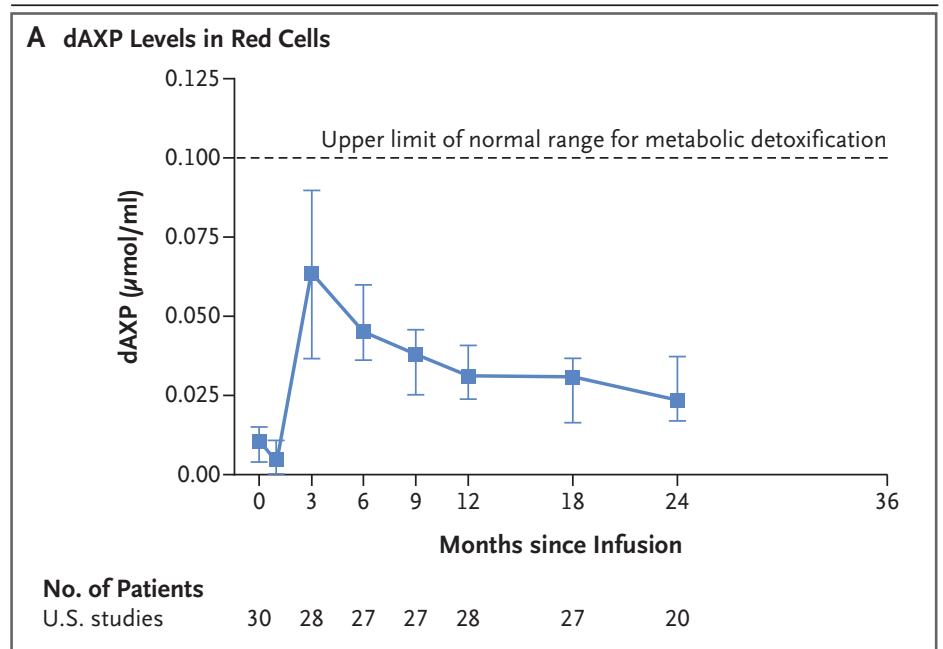

B dATP Levels in Red Cells

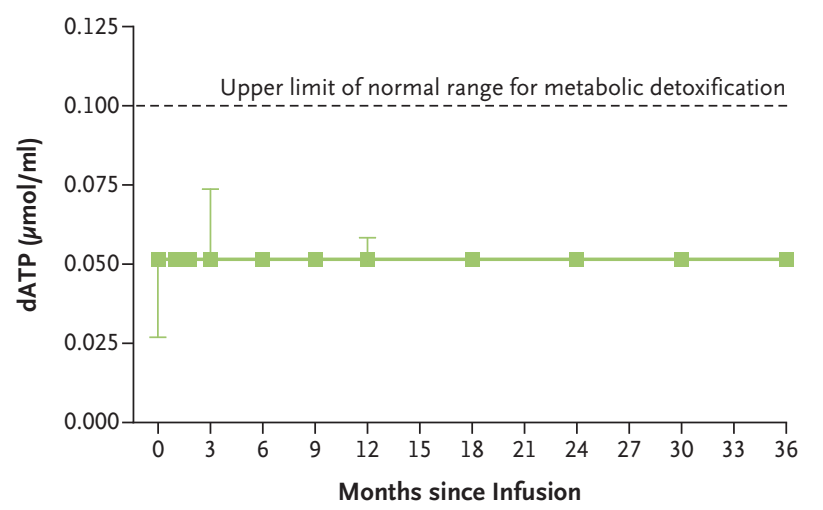

No. of Patients

$\begin{array}{llllllllll}\text { U.K. study } & 17 & 16 & 16 & 17 & 14 & 18 & 15 & 14 & 15\end{array}$

Figure 3. Median dAXP and dATP Levels in Red Cells.

Levels of deoxyadenosine metabolites measured in red cells by high-pressure liquid chromatography are shown. Panel A shows median total deoxyadenosine nucleotide (dAXP) (including deoxyadenosine monophosphate, deoxyadenosine diphosphate, and deoxyadenosine triphosphate [dATP]) levels, and Panel B shows median dATP purine metabolite levels. In both panels, I bars indicate interquartile ranges. The dashed line indicates the threshold below which patients are considered to have adequate detoxification. 


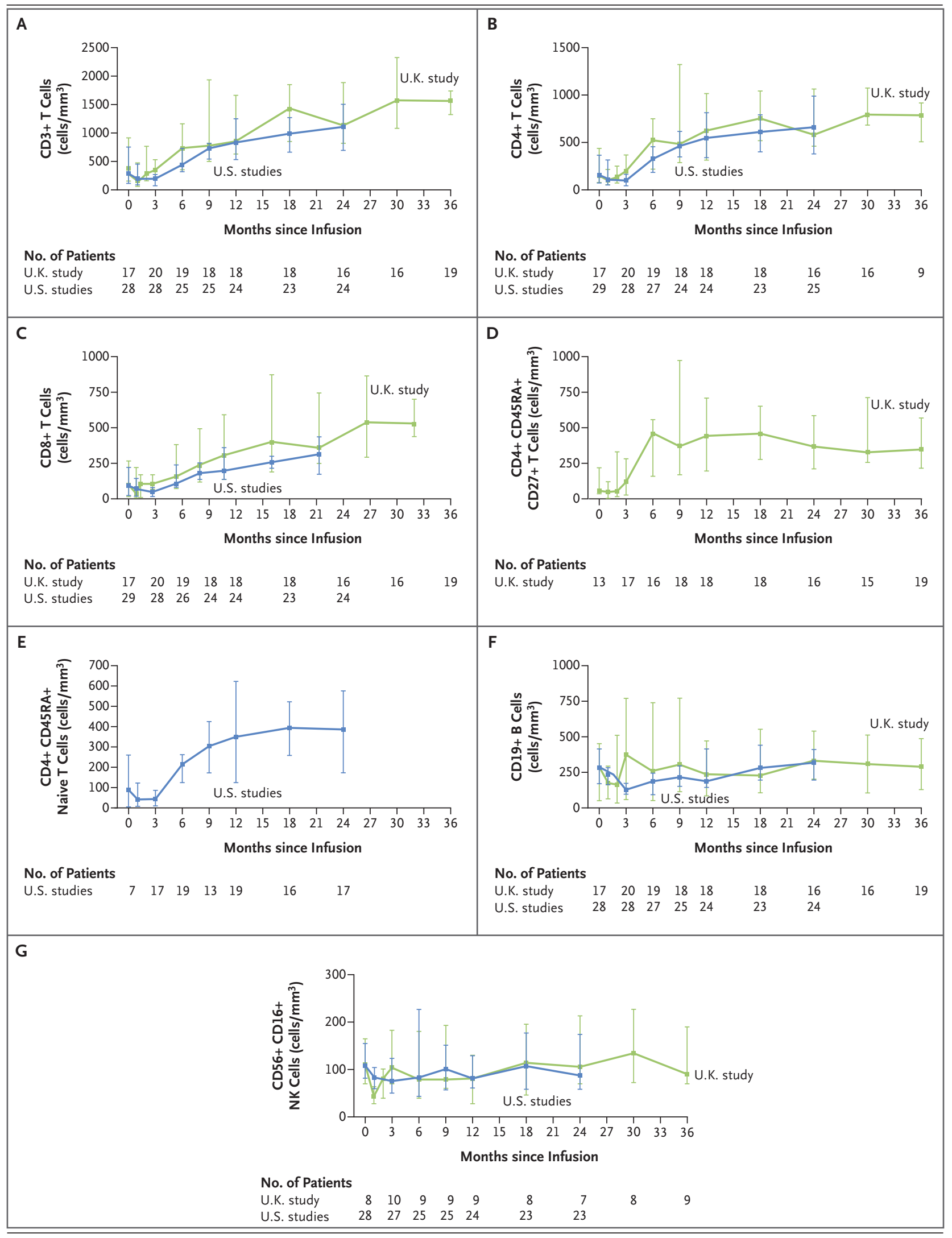


Figure 4 (facing page). Median Absolute Lymphocyte Counts as Determined by Flow Cytometry.

Median absolute lymphocyte counts over 24 or 36 months are shown. In all panels, I bars indicate interquartile ranges. NK denotes natural killer.

Across the three studies, severe infection rates were generally low after gene therapy (Fig. S7). Fifteen serious infections were observed in 8 patients in the U.S. studies, including bronchiolitis, upper respiratory tract infection, pneumonia, bacteremia, Clostridium difficile colitis, device-related infection, influenza, otitis media, respiratory tract infection (viral), and rhinovirus infection. Eight serious infections were observed in 7 patients in the U.K. study, including central catheter sepsis, norovirus-related gastroenteritis, influenza, pneumonia, transmission of coagulase-negative staphylococcus (from known drugproduct contamination), urinary tract infection, and oral candidiasis.

Efficacy results in U.S. patients who received the fresh formulation were similar to those in patients who received the cryopreserved formulation, as indicated by the median vector copy number in granulocytes and PBMCs, median $\mathrm{CD} 3+$ T-cell levels, and median ADA activity (Fig. S8).

\section{DISCUSSION}

All the patients in these three studies were alive and well at last follow-up, with no reports of fatal or life-threatening adverse events, clonal expansions, or leukoproliferative complications. Evidence of sustained immune reconstitution and thymic recovery was clear in most patients, and the large majority of patients were no longer receiving immunoglobulin-replacement therapy by the end of the study. Severe infection rates were highest in the first 3 months after treatment, as expected because of conditioning, but were generally low across the full post-treatment period in all studies.

Overall and event-free survival (100\% and $>95 \%$, respectively) at 12, 24, and 36 months compare favorably with those among patients undergoing HSCT, the current standard of care. Overall survival of 65 to $88 \%$ has been reported among patients receiving HSCT from matched and unmatched donors, and event-free survival has been reported to be $56 \% .{ }^{23,26,27}$ A U.S. study also reported 2-year survival of $100 \%$ among 28 patients receiving gene therapy as compared with $87 \%$ among the 40 patients who received HSCT. ${ }^{28}$

The predominantly uncomplicated clinical course we observed may be attributed to the low busulfan dose used for conditioning. The most notable complication was the development of immune reconstitution inflammatory syndrome in 4 patients, all of whom had response to treatment with glucocorticoids. Only two treatment failures were observed across all studies, with 1 U.K. patient and 1 U.S. patient being withdrawn from their studies and having enzyme-replacement therapy reinitiated.

In a recent consensus report, HSCT from a matched related donor and gene therapy were referred to as equivalent first-line options for the treatment of ADA-SCID. ${ }^{4}$ The absence of graftversus-host disease and the obviation of immunosuppressive therapies are major potential advantages of autologous HSPC gene therapy. None of the patients described here had evidence of replication-competent lentivirus or clonal expansions, which adds to the growing evidence for the safety of lentiviral vectors that has been seen in other clinical studies, ${ }^{29-36}$ in which various genes have been introduced in patients with other disorders, with follow-up extending to more than 10 years for the first patients treated. Other potential advantages of gene therapy over allogeneic HSCT include the absence of a donor requirement and lower toxicity, since less conditioning is required. Furthermore, as compared with HSCT, in which enzyme-replacement therapy is discontinued before treatment in order to limit the expansion of host $\mathrm{T}$ cells and the potential for graft-versus-host disease, a gene-therapy approach allows for continuation of enzymereplacement therapy during immune reconstitution and thus for continued protection against infections during this period.

Autologous CD34+ HSPCs were successfully transduced with EFS-ADA LV for all patients. After infusion, sustained levels of gene marking in leukocytes, including short-lived granulocytes, were present, a finding that suggests successful and stable engraftment of genetically modified long-term repopulating HSPCs. The increase in vector copy number in PBMCs, including lymphocytes, indicates the accumulation of genetically modified cells and suggests that a selective survival advantage was conferred on mature ge- 
netically modified lymphocytes carrying the functional $A D A$ transgene.

Restored CD19+ B-cell counts and immunoglobulin production indicate restoration of the humoral immune system. In the U.S. studies, $89.7 \%$ of the patients stopped receiving immunoglobulin-replacement therapy by 24 months (end of study), and $100 \%$ of the patients in the U.K. study stopped by 36 months (end of study). The treating physicians have reported that all the patients in the U.S. studies are now no longer receiving immunoglobulin-replacement therapy and that all the patients in the U.K. study have successfully received tetanus vaccinations.

In this report, we have presented the results from three separate clinical studies involving distinct populations of patients with ADA-SCID in the United States and the United Kingdom. Although the same lentiviral vector (EFS-ADA LV) was used in all three studies, differences among the studies included different manufacturing processes, cell sources (HSPCs were harvested from bone marrow in the U.S. studies and predominantly from mobilized peripheral blood in the U.K. study), and final drug-product formulations (the U.S. studies used either fresh or cryopreserved formulations and the U.K. study used a fresh formulation). With a cryopreserved formulation, all release testing is conducted and certificates of analysis issued before administration of the conditioning regimen and administration of the drug product. Similar outcomes were observed regardless of the cell source and formulation.

The patient populations also spanned a broad age range. The U.K. study included five patients older than 5 years of age, three of whom were 10 years of age or older $(10,11$, and 16 years) and had been receiving enzyme-replacement therapy for more than 10 years. Thus, gene therapy with HSPCs that have been genetically modified ex vivo with EFS-ADA LV may be a robust and viable treatment option for patients with ADA-SCID, including older children for whom current treatments are not always suitable or effective and who may have reduced or absent thymic function.

Sustained engraftment of genetically modified cells resulted in restored ADA enzyme activity and immune system function in 48 of 50 patients with ADA-SCID who were treated with gene therapy consisting of HSPCs transduced ex vivo with a lentiviral vector. The high overall survival and event-free survival observed at 24 and 36 months compare favorably with survival that has been observed previously with HSCT, the current standard of care for the treatment of ADA-SCID.

Supported by grants (U01 AI100801, 2P01 HL073104, and National Heart, Lung, and Blood Institute [NHLBI] Gene Therapy Resource Program Request for Service applications 1101 and 1129) from the National Institutes of Health (NIH), the National Human Genome Research Institute intramural program, the National Institute of Allergy and Infectious Diseases (NIAID) intramural program, the National Gene Vector Biorepository (NHLBI contract 75N92019D00018), grants (CLIN2-09339, CL100505, FA1-00613, and AC1-07675) from the California Institute for Regenerative Medicine, grants (MR/K015427/1 and G80248) from the Medical Research Council, a grant (AC1-07675) from the National Institute for Health Research Biomedical Research Centre at the Great Ormond Street Hospital for Children NHS Foundation Trust and University College London, and Orchard Therapeutics (Europe). Dr. Booth is supported by the Mahboubian family and Great Ormond Street Hospital Children's Charity. Dr. Thrasher is a Wellcome Trust Principal Research Fellow (grant 217112/Z/19/Z).

Disclosure forms provided by the authors are available with the full text of this article at NEJM.org.

A data sharing statement provided by the authors is available with the full text of this article at NEJM.org.

We thank Dr. Linda Griffith for support and encouragement for the implementation of the gene-therapy study; the patients and their families and their home doctors and nurses; the physicians who referred the patients who received gene therapy in the United States and participated in their care and evaluations: Dr. Rajni Agarwal, Dr. Scott Cameron, Dr. Karin Chen, Dr. Geoffrey Cuvelier, Dr. Blachy Davila, Dr. Maite de la Morena, Dr. Morna Dorsey, Dr. Jose Luis Franco, Dr. Eyal Grunebaum, Dr. Elie Haddad, Dr. Andrew Harris, Dr. Nabil Kabbara, Dr. Neena Kapoor, Dr. Alan Knutsen, Dr. Lisa Kobrynski, Dr. Howard Lederman, Dr. Rebecca Marsh, Dr. Luis Murguia-Favela, Dr. Sung-Yun Pai, Dr. Anne Pham-Huey, Dr. Jennifer Puck, Dr. Marina Salvadori, Dr. Kirk Schultz, Dr. Evan Shereck, Dr. John Sleasman, Dr. Angela Smith, Dr. Elizabeth Stenger, and Dr. Kenneth I. Weinberg; Dr. Suk See De Ravin and Dr. Alan Wayne, for their participation in clinical care at the NIH; the University of California, Los Angeles (UCLA), David Geffen School of Medicine Human Gene and Cell Therapy Program, the UCLA Eli and Edythe Broad Center of Regenerative Medicine and Stem Cell Research, and the UCLA Jonsson Comprehensive Cancer Center, for provision of resources for the study; the physicians who referred the patients who received gene therapy in the United Kingdom and participated in their care and evaluations: Dr. Despina Moshous, Dr. Benedicte Neven, Dr. Nizar Mahlaoui, Dr. Tore Abrahamsen, Dr. Torstein Фverland, Dr. David Moreno Perez, Dr. Rosie Hague, Dr. Scott Hackett, Dr. Vicky Bordon, Dr. Carsten Speckmann, Dr. Terry Flood, Dr. Michael Gold, Dr. Jens Erik Veirum, Dr. Troels Herlin, Dr. Mette Holm, Dr. Ronan Leahy, and Dr. Paul Veys; Phastar, under the supervision of Ms. Frances Lynn (Orchard Therapeutics [Europe]), for performing the statistical analyses; Dr. Robin LeWinter and Dr. Cindy Chen from Orchard Therapeutics (Europe) for their help coordinating the writing of an earlier version of the manuscript; and Dr. Lara Bennett and Dr. Ben Drever (Comradis, U.K.) for editorial support, paid for by Orchard Therapeutics, with an earlier version of the manuscript. 
APPENDIX

The authors' full names and academic degrees are as follows: Donald B. Kohn, M.D., Claire Booth, M.B., B.S., Kit L. Shaw, Ph.D., Jinhua Xu-Bayford, D.I.P., Elizabeth Garabedian, R.N., Valentina Trevisan, M.D., Denise A. Carbonaro-Sarracino, Ph.D., Kajal Soni, B.Sc., Dayna Terrazas, R.N., Katie Snell, B.Sc., Alan Ikeda, M.D., Diego Leon-Rico, Ph.D., Theodore B. Moore, M.D., Karen F. Buckland, Ph.D., Ami J. Shah, M.D., Kimberly C. Gilmour, Ph.D., Satiro De Oliveira, M.D., Christine Rivat, Ph.D., Gay M. Crooks, M.B., B.S., Natalia Izotova, B.Sc., John Tse, Pharm.D., Stuart Adams, Ph.D., Sally Shupien, B.A., Hilory Ricketts, B.Sc., Alejandra Davila, B.S., Chilenwa Uzowuru, M.Sc., Amalia Icreverzi, Ph.D., Provaboti Barman, Ph.D., Beatriz Campo Fernandez, Ph.D., Roger P. Hollis, Ph.D., Maritess Coronel, M.S., Allen Yu, B.S., Krista M. Chun, B.S., Christian E. Casas, B.S., Ruixue Zhang, Ph.D., Serena Arduini, Ph.D., Frances Lynn, M.Sc., Mahesh Kudari, M.B., B.S., Andrea Spezzi, M.D., Marco Zahn, M.Sc., Rene Heimke, M.Sc., Ivan Labik, M.Sc., Roberta Parrott, B.S., Rebecca H. Buckley, M.D., Lilith Reeves, M.S., Kenneth Cornetta, M.D., Robert Sokolic, M.D., Michael Hershfield, M.D., Manfred Schmidt, Ph.D., Fabio Candotti, M.D., Harry L. Malech, M.D., Adrian J. Thrasher, M.B., B.S., and H. Bobby Gaspar, M.B., B.S.

The authors' affiliations are as follows: the Departments of Microbiology, Immunology, and Molecular Genetics (D.B.K., K.L.S., D.A.C.-S., D.T., A.D., A. Icreverzi, P.B., B.C.F., R.P.H., M.C., A.Y., K.M.C., C.E.C., R.Z.), Pediatrics (D.B.K., T.B.M., S.D.O., S.S.), and Pathology and Laboratory Medicine (G.M.C.) and the Eli and Edythe Broad Center of Regenerative Medicine and Stem Cell Research (D.B.K., G.M.C.), University of California, Los Angeles (UCLA), and the Department of Pharmaceutical Services, Ronald Reagan UCLA Medical Center (J.T.), Los Angeles, and Stanford School of Medicine, Palo Alto (A.J.S.) - all in California; University College London Great Ormond Street Institute of Child Health and Great Ormond Street Hospital for Children NHS Foundation Trust (C.B., J.X.-B., V.T., K. Soni, K. Snell, D.L.-R., K.F.B., K.C.G., C.R., N.I., S.A., H.R., C.U., A.J.T., H.B.G.), and Orchard Therapeutics (Europe) (D.A.C.S., S.A., F.L., M.K., A.S., H.B.G.) - all in London; the National Institute of Allergy and Infectious Diseases (H.L.M.) and the National Human Genome Research Institute (E.G., R.S., F.C.), National Institutes of Health, Bethesda, MD; Cure 4 The Kids Foundation, Las Vegas (A. Ikeda); Cincinnati Children's Hospital Medical Center, Cincinnati (L.R.); Indiana University School of Medicine, Indianapolis (K.C.); Duke University, Durham, NC (R.P., R.H.B., M.H.); Division of Immunology and Allergy, Lausanne University Hospital and University of Lausanne, Lausanne, Switzerland (F.C.); and GeneWerk (M.Z., R.H., I.L., M.S.) and the German Cancer Research Center and the National Center for Tumor Diseases (M.Z., M.S.) — all in Heidelberg, Germany.

\section{REFERENCES}

1. Hirschhorn R. Adenosine deaminase deficiency: molecular basis and recent developments. Clin Immunol Immunopathol 1995;76:S219-S227.

2. Sauer AV, Brigida I, Carriglio N, Aiuti A. Autoimmune dysregulation and purine metabolism in adenosine deaminase deficiency. Front Immunol 2012;3:265.

3. Hershfield MS. Adenosine deaminase deficiency: clinical expression, molecular basis, and therapy. Semin Hematol 1998; 35:291-8.

4. Kohn DB, Hershfield MS, Puck JM, e al. Consensus approach for the management of severe combined immune deficiency caused by adenosine deaminase deficiency. J Allergy Clin Immunol 2019; 143:852-63.

5. EBMT/ESID guidelines for haematopoietic stem cell transplantation for primary immunodeficiencies. European Society for Blood and Marrow Transplantation, 2017 (https://www.ebmt.org/sites/ default/files/migration_legacy_files/ document/Inborn\%20Errors\%20Working \%20Party\%20ESID\%20EBMT\%20 HSCT\%20Guidelines\%202017.pdf).

6. Booth C, Gaspar HB. Pegademase bovine (PEG-ADA) for the treatment of infants and children with severe combined immunodeficiency (SCID). Biologics 2009; 3:349-58.

7. Chan B, Wara D, Bastian J, et al. Longterm efficacy of enzyme replacement therapy for adenosine deaminase (ADA)deficient severe combined immunodeficiency (SCID). Clin Immunol 2005;117: 133-43.

8. Ferrua F, Brigida I, Aiuti A. Update on gene therapy for adenosine deaminasedeficient severe combined immunodeficiency. Curr Opin Allergy Clin Immunol 2010;10:551-6.

9. Haddad E, Landais P, Friedrich W, et al. Long-term immune reconstitution and outcome after HLA-nonidentical T-celldepleted bone marrow transplantation for severe combined immunodeficiency: a European retrospective study of 116 patients. Blood 1998;91:3646-53.

10. Neven B, Leroy S, Decaluwe H, et al. Long-term outcome after hematopoietic stem cell transplantation of a single-center cohort of 90 patients with severe combined immunodeficiency. Blood 2009; 113:4114-24.

11. Aiuti A, Cattaneo F, Galimberti S, et al. Gene therapy for immunodeficiency due to adenosine deaminase deficiency. N Engl J Med 2009;360:447-58.

12. Cicalese MP, Ferrua F, Castagnaro L, et al. Update on the safety and efficacy of retroviral gene therapy for immunodeficiency due to adenosine deaminase deficiency. Blood 2016;128:45-54.

13. Strimvelis. Strimvelis European Medicines Agency, 2016 (https://www.ema .europa.eu/en/medicines/human/EPAR/ strimvelis\#authorisation-details-section). 14. Hacein-Bey-Abina S, Von Kalle C, Schmidt M, et al. LMO2-associated clonal $\mathrm{T}$ cell proliferation in two patients after gene therapy for SCID-X1. Science 2003; 302:415-9.

15. Howe SJ, Mansour MR, Schwarzwaelder $\mathrm{K}$, et al. Insertional mutagenesis combined with acquired somatic mutations causes leukemogenesis following gene therapy of SCID-X1 patients. J Clin Invest 2008;118:3143-50.

16. Stein S, Ott MG, Schultze-Strasser S, et al. Genomic instability and myelodysplasia with monosomy 7 consequent to EVI1 activation after gene therapy for chronic granulomatous disease. Nat Med 2010;16:198-204.

17. Orchard statement on Strimvelis, a gammaretroviral vector-based gene therapy for ADA-SCID. Orchard therapeutics. October 30, 2020 (https://ir.orchard-tx .com/news-releases/news-release-details/ orchard-statement-strimvelisr -gammaretroviral-vector-based-gene).

18. Carbonaro DA, Zhang L, Jin X, et al. Preclinical demonstration of lentiviral vector-mediated correction of immunological and metabolic abnormalities in models of adenosine deaminase deficiency. Mol Ther 2014;22:607-22.

19. Montiel-Equihua CA, Zhang L, Knight $\mathrm{S}$, et al. The $\beta$-globin locus control region in combination with the EF $1 \alpha$ short promoter allows enhanced lentiviral vectormediated erythroid gene expression with conserved multilineage activity. Mol Ther 2012;20:1400-9.

20. Zychlinski D, Schambach A, Modlich $\mathrm{U}$, et al. Physiological promoters reduce the genotoxic risk of integrating gene vectors. Mol Ther 2008;16:718-25.

21. Zufferey R, Dull T, Mandel RJ, et al. Self-inactivating lentivirus vector for safe and efficient in vivo gene delivery. J Virol 1998;72:9873-80.

22. Muraoka T, Katsuramaki T, Shiraishi H, Yokoyama MM. Automated enzymatic measurement of adenosine deaminase 
isoenzyme activities in serum. Anal Biochem 1990;187:268-72.

23. Hassan A, Booth C, Brightwell A, et al. Outcome of hematopoietic stem cell transplantation for adenosine deaminasedeficient severe combined immunodeficiency. Blood 2012;120:3615-24.

24. Gaspar HB, Aiuti A, Porta F, Candotti F, Hershfield MS, Notarangelo LD. How I treat ADA deficiency. Blood 2009;114: 3524-32.

25. Griffith LM, Cowan MJ, Notarangelo LD, et al. Improving cellular therapy for primary immune deficiency diseases: recognition, diagnosis, and management. J Allergy Clin Immunol 2009;124(6):11521160.e12.

26. Kuo CY, Garabedian E, Puck J, et al. Adenosine deaminase (ADA)-deficient severe combined immune deficiency (SCID) in the US Immunodeficiency Network (USIDNet) registry. J Clin Immunol 2020; 40:1124-31.

27. Kohn DB, Shaw KL, Garabedian E, et al. Lentiviral gene therapy with autologous hematopoietic stem and progenitor cells (HSPCs) for the treatment of severe combined immune deficiency due to adenosine deaminase deficiency (ADA-SCID) two year follow-up results. Mol Ther 2020;28:Suppl 1:1300. abstract.

28. Kuo CY, Puck JM, Logan BR, et al. Adenosine deaminase (ADA)-deficient severe combined immune deficiency (SCID): analysis of cases enrolled in protocols of the Primary Immune Deficiency Treatment Consortium (PIDTC). J Clin Immunol 2018;38:342-3. abstract.

29. Biffi A, Montini E, Lorioli L, et al Lentiviral hematopoietic stem cell gene therapy benefits metachromatic leukodystrophy. Science 2013;341:1233158.

30. Cartier N, Hacein-Bey-Abina S, Bartholomae CC, et al. Hematopoietic stem cell gene therapy with a lentiviral vecto in X-linked adrenoleukodystrophy. Science 2009;326:818-23.

31. Eichler F, Duncan C, Musolino PL, et al. Hematopoietic stem-cell gene therapy for cerebral adrenoleukodystrophy. N Engl J Med 2017;377:1630-8.

32. Ferrua F, Cicalese MP, Galimberti S, et al. Lentiviral haemopoietic stem/progenitor cell gene therapy for treatment of Wiskott-Aldrich syndrome: interim results of a non-randomised, open-label, phase $1 / 2$ clinical study. Lancet Haematol 2019;6(5):e239-e253.

33. Kohn DB, Booth C, Kang EM, et al. Lentiviral gene therapy for X-linked chronic granulomatous disease. Nat Med 2020; 26:200-6.

34. Marktel S, Scaramuzza S, Cicalese $\mathrm{MP}$, et al. Intrabone hematopoietic stem cell gene therapy for adult and pediatric patients affected by transfusion-dependent $\beta$-thalassemia. Nat Med 2019;25:234-41.

35. Sessa M, Lorioli L, Fumagalli F, et al. Lentiviral haemopoietic stem-cell gene therapy in early-onset metachromatic leukodystrophy: an ad-hoc analysis of a nonrandomised, open-label, phase 1/2 trial. Lancet 2016;388:476-87.

36. Thompson AA, Walters MC, Kwiatkowski J, et al. Gene therapy in patients with transfusion-dependent beta-thalassemia. N Engl J Med 2018;378:1479-93.

Copyright (๑) 2021 Massachusetts Medical Society. 